\title{
Response Dissociation in Hierarchical Cortical Circuits: a Unique Feature of Autism Spectrum Disorder
}

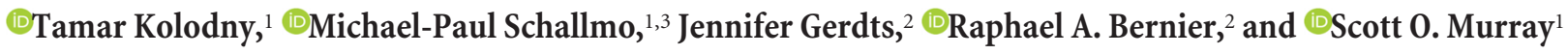 \\ Departments of ${ }^{1}$ Psychology, ${ }^{2}$ Psychiatry and Behavioral Sciences, University of Washington, Seattle, Washington 95195 , and ${ }^{3}$ Department of Psychiatry \\ and Behavioral Science, University of Minnesota, Minneapolis, Minnesota 55455
}

A prominent hypothesis regarding the pathophysiology of autism is that an increase in the balance between neural excitation and inhibition results in an increase in neural responses. However, previous reports of population-level response magnitude in individuals with autism have been inconsistent. Critically, network interactions have not been considered in previous neuroimaging studies of excitation and inhibition imbalance in autism. In particular, a defining characteristic of cortical organization is its hierarchical and interactive structure; sensory and cognitive systems are comprised of networks where later stages inherit and build upon the processing of earlier input stages, and also influence and shape earlier stages by top-down modulation. Here we used the well established connections of the human visual system to examine response magnitudes in a higher-order motion processing region [middle temporal area $(\mathrm{MT}+)$ ] and its primary input region (V1). Simple visual stimuli were presented to adult individuals with autism spectrum disorders (ASD; $n=$ 24, mean age 23 years, 8 females) and neurotypical controls ( $n=24$, mean age 22, 8 females) during fMRI scanning. We discovered a strong dissociation of fMRI response magnitude between region MT + and V1 in individuals with ASD: individuals with high MT+ responses had attenuated V1 responses. The magnitude of MT + amplification and of V1 attenuation was associated with autism severity, appeared to result from amplified suppressive feedback from MT + to $\mathrm{V} 1$, and was not present in neurotypical controls. Our results reveal the potential role of altered hierarchical network interactions in the pathophysiology of ASD.

Key words: autism; E/I balance; fMRI; visual cortex

\section{Significance Statement}

An imbalance between neural excitation and inhibition, resulting in increased neural responses, has been suggested as a pathophysiological pathway to autism, but direct evidence from humans is lacking. In the current study we consider the role of interactions between stages of sensory processing when testing increased neural responses in individuals with autism. We used the well known hierarchical structure of the visual motion pathway to demonstrate dissociation in the fMRI response magnitude between adjacent stages of processing in autism: responses are attenuated in a primary visual area but amplified in a subsequent higher-order area. This response dissociation appears to rely on enhanced suppressive feedback between regions and reveals a previously unknown cortical network alteration in autism.

\section{Introduction}

Autism spectrum disorder (ASD) is a heterogeneous, behaviorallydefined neurodevelopmental disorder characterized by difficulties in social communication and interaction, restricted interests

\footnotetext{
Received Oct. 2, 2019; revised Jan. 11, 2020; accepted Jan. 22, 2020.

Author contributions: T.K., R.A.B., and S.O.M. designed research; T.K., M.-P.S., and J.G. performed research; T.K., M.P.S., and S.O.M. analyzed data; T.K. wrote the paper.

This work was supported by funding from the National Institutes of Health (R01 MH106520 to S.0.M.) and by the Israel Science Foundation (Grant 103/19 to T.K.). We thank Rachel Millin, Alex Kale, Anastasia Flevaris, Ly Nguyen, Heena Panjwani, Micah Pepper, and the University of Washington Diagnostic Imaging Center for help with recruitment, data collection, and/or data analysis.

The authors declare no competing financial interests.

Correspondence should be addressed to Tamar Kolodny at tamark@uw.edu.

https://doi.org/10.1523/JNEUROSCI.2376-19.2020

Copyright $\odot 2020$ the authors
}

and repetitive behaviors, and altered sensory responses (DSM-5; American Psychiatric Association, 2013; Lord and Bishop, 2015). Although the etiology of ASD is unknown, a pervasive increase in the ratio of cortical excitation to inhibition (E/I) has been hypothesized to be a primary contributor to the underlying pathophysiology, resulting in neuronal hyperexcitability (Rubenstein and Merzenich, 2003; Nelson and Valakh, 2015). Indeed, animal model findings support the E/I model of ASD (Gogolla et al., 2009; Yizhar et al., 2011; Antoine et al., 2019), and a causal relationship to the autism phenotype has been suggested using pharmacology and gene-rescue approaches that alter E/I balance (Han et al., 2012; Lee et al., 2017). However in humans, evidence for E/I imbalance is either indirect or inconclusive in its support of this hypothesis (Dickinson et al., 2016). One straightforward predic- 
tion of an increase in $\mathrm{E} / \mathrm{I}$ are higher neural spike rates (Contractor et al., 2015). Consequently, larger amplitude neural population responses are expected, which could be indexed with fMRI and/or EEG. However, there are no consistent demonstrations of such increased population-based responses in individuals with ASD (Milne, 2011; Dinstein et al., 2012; Haigh et al., 2015; Butler et al., 2017).

A complicating factor that has recently been recognized for the $\mathrm{E} / \mathrm{I}$ hypothesis is the homeostatic regulatory mechanisms that adjust $\mathrm{E} / \mathrm{I}$ balance to stabilize cortical firing rate (Turrigiano, 2011); thus, increases or decreases in excitation in one component of a neural circuit may be compensated for at a later component (Nelson and Valakh, 2015), and ASD may be characterized by suboptimal homeostasis causing interruption in population coding (Antoine et al., 2019). Similarly, on a larger spatial scale, interactions between cortical areas involved in different stages of information processing may also involve regulatory and/or compensatory mechanisms, and such interactions are further complicated given the recurrent structure of cortical networks (Mejias et al., 2016; Heeger, 2017). For example, the feedforward, hierarchical organization of sensory systems is complemented by prominent feedback projections, further shaping neural activity by top-down modulation (Felleman and Van Essen, 1991; Sillito et al., 2006; Gilbert and Li, 2013), which can serve to amplify or suppress responses (Bullier et al., 1996; Hupé et al., 1998; Bair et al., 2003; Angelucci and Bressloff, 2006). This type of network interaction has not been previously considered when examining populationlevel responses in ASD, and might have been obscuring group differences.

In the current study we specifically leveraged the well established hierarchical structure of the visual motion processing pathway, to measure fMRI response magnitude in adjacent stages of cortical processing. Atypical visual motion processing has been reported in autism using various stimuli and tasks, with some reports of reduced sensitivity (Milne et al., 2002; Pellicano et al., 2005; Robertson et al., 2012; Schauder et al., 2017) and others of enhanced sensitivity (Chen et al., 2012; Foss-Feig et al., 2013; Manning et al., 2015). While the nature of these atypicalities and how they depend on the type and presentation of motion stimuli is yet to be clarified, the visual motion processing pathway is a good candidate to characterize neural responsiveness within a well characterized cortical system that is also hypothesized to be altered in ASD, and possibly relate to relevant perceptual patterns (Robertson et al., 2014).

We show that although neural response magnitude is indeed altered in ASD, this alteration is not unidirectional along the visual motion pathway, and might take the form of either increased or decreased responses. We suggest this mixed pattern of results stems from inter-regional interactions and abnormal cortical feedback in ASD, and that such interactions should be incorporated with the basic E/I model to inform testable predictions for $\mathrm{E} / \mathrm{I}$ in the human brain.

\section{Materials and Methods}

Participants. Twenty-four adults with ASD (16 males; 8 females) and 24 neurotypical (NT; 16 males; 8 females) young adults participated in Experiment 1 . The same cohorts also participated in other studies recently reported by our group (Millin et al., 2018; Schallmo et al., 2019a); and NT participants were also included in (Murray et al., 2018; Schallmo et al., 2018, 2019b). All participants had normal IQ (Wechsler Abbreviated Scale of Intelligence score; Stano, 2004) of at least 80, and normal or corrected-to-normal vision. Groups were of equivalent ages and IQ [mean non-verbal IQ of ASD participants: 112.5 (SD = 18.8; range 79$160)$; NT participants: $113.8(\mathrm{SD}=10.5$; range $86-129)$; $t_{(46)}=-0.28$, $p=0.77$; mean age of ASD participants: $22.7(\mathrm{SD}=3.7$; range 18-31) years; NT participants: 22.3 ( $\mathrm{SD}=2.6$; range $19-28)$ years; $t_{(46)}=0.45$, $p=0.66]$. All participants reported smoking no more than one cigarette per day within the past 3 months, no illicit drug use within the past month, and no alcohol use within $3 \mathrm{~d}$ before scanning. Participants with ASD met diagnostic criteria for ASD on the Autism Diagnostic InterviewRevised (ADI-R; Lord et al., 1994), the Autism Diagnostic Observation Schedule, second edition (ADOS-2; Lord et al., 2012) and according to expert clinical judgment using DSM-5 (American Psychiatric Association, 2013) criteria. The ADOS-2 Overall Comparison Score, which reports ASD symptom severity on a scale from 1 to 10 , was calculated and used in correlational analyses. Participants were included in the study only if stable on any medication dosage for at least 3 months before the study. Of these participants in Experiment 1, 11 participants with ASD ( 9 males, 2 females), 7 NT participants ( 5 males, 2 females) and one additional NT male participant also attended Experiment 2. Two ASD participants and one NT participant were excluded before analysis because of observed head motion and low data quality in the scanner, resulting in a final sample of 9 ASD participants and 7 NT participants in Experiment 2. All participants provided written informed consent to participate, and were monetary compensated for their time. The research protocol was approved by the Institutional Review Board of the University of Washington.

MRI acquisition. Data were acquired using a Philips Achieva 3-tesla scanner with a 32-channel high-resolution head coil. T1-weighted MPRAGE structural MRI data were acquired in each session at $1 \mathrm{~mm}$ isotropic resolution. Functional MRI data (gradient echo EPI) were acquired with $3 \times 3 \mathrm{~mm}$ in-plane resolution. Thirty oblique-axial slices were obtained ( $3 \mathrm{~mm}$ slice thickness, separated by a $0.5 \mathrm{~mm}$ gap). Other scan parameters: $2 \mathrm{~s} \mathrm{TR,} 25 \mathrm{~ms} \mathrm{TE}, 79^{\circ}$ flip angle, A-P phase-encode direction. At the start of each session, an opposite phase-encode direction (P-A) scan (1 TR) was acquired for distortion compensation. Each subject underwent two 1-h-long scanning sessions for Experiment 1, each including primary visual cortex (V1) and middle temporal area $(\mathrm{MT}+)$ functional localizer scans and two experimental scans. This experiment was performed as part of a larger set of visual fMRI experiments focused on the neural mechanisms of surround suppression in ASD, additional functional scans that were included in the sessions are reported elsewhere (Schallmo et al., 2019a). Experiment 2 took place between 1 and 3 years after completion of Experiment 1 (mean time difference between experiments for each participant: 647 d, SD = 292). Participants completed one (NT) or two (ASD) 1-h-long scanning sessions in this experiment. During all scanning sessions, subjects' eye movements were recorded using an EyeLink 1000 Plus eye-tracker, sampling at $1000 \mathrm{~Hz}$.

Stimuli and task design. Stimuli were displayed via projector (Epson Powerlite 7250 or Eiki LCXL100A, following a hardware failure), operating at $60 \mathrm{~Hz}$ using Presentation software v14.9 (Neurobehavioral Systems) on a PC running Windows XP. Images were projected on a semicircular screen at the rear of the scanner, and viewed through a mirror on the head coil at a distance of $66 \mathrm{~cm}$. Projector luminance was linearized using custom software.

Experimental stimuli were similar to those described previously (Schallmo et al., 2018). Briefly, drifting sinusoidal luminance modulated gratings at two different Michelson contrast levels (low $=3 \%$, high $=$ $98 \%$ ) were used, with a spatial frequency of $1 \mathrm{cycle} /{ }^{\circ}$ and a drift rate of 4 cycle/s. Gratings were presented within a circular window, whose edges were blurred with a Gaussian envelope $\left(\mathrm{SD}=0.25^{\circ}\right)$. In Experiment 1 stimuli diameter was $2^{\circ}$ visual angle, and they were presented in the center of the screen. In Experiment 2, four gratings were presented simultaneously in the four quadrants of the visual field, each subtending $3^{\circ}$ visual angle diameter and centered at an eccentricity of $5^{\circ}$. In both experiments grating orientations $\left(0^{\circ}, 45^{\circ}, 90^{\circ}, 135^{\circ}\right)$ and drifting directions changed after a presentation duration of $400 \mathrm{~ms}$, with an interstimulus interval of $267 \mathrm{~ms}$. Different orientations and motion directions appeared with equal prevalence within each block, in a randomized order. Blocks of drifting gratings alternated with blank background blocks, with drifting gratings blocks alternating between high and low contrast gratings. Each block was $10 \mathrm{~s}$ long, with a total of 25 blocks ( 6 high contrast, 6 low contrast, 13 blank). Each participant completed between 2 and 4 
experimental scans across 1-2 scanning sessions in each of the experiments (some scans were cut short or canceled due to time constraints or participants discomfort).

Functional localizer scans were acquired to identify regions-of-interest (ROIs), with designs closely resembling that of the experimental scans, and with stimuli in the same retinotopic locations. To identify areas V1, $\mathrm{V} 2$, and V3 in the visual cortex, the stimuli used were circular $100 \%$ contrast checkerboards phase-reversing at $8 \mathrm{~Hz}$, in the same size and location/s as the experimental stimuli (i.e., $2^{\circ}$ diameter in the center of the screen in Experiment $1,3^{\circ}$ diameter centered $5^{\circ}$ from fixation in Experiment 2). Blocks with flickering checkerboards alternated with blank background blocks, each block $10 \mathrm{~s}$ long, for a total of 16 blocks per scan. A second localizer was used to identify human MT complex $(\mathrm{MT}+)$; we use this annotation to clarify that we did not attempt to distinguish between MT and medial superior temporal area (MST), both of which are motion selective (Huk et al., 2002). Stimuli were sinusoidal luminance modulated gratings at $15 \%$ contrast. Stimuli were the same size and had the same spatial frequency as the experimental stimuli, and were presented in the same retinotopic locations (at fixation in Experiment $1,5^{\circ}$ eccentricity in Experiment 2), without edge blurring. Blocks of drifting gratings $\left(4 \mathrm{cycle} /{ }^{\circ}\right.$, same drift rate of the experimental stimuli) alternated with blocks of static gratings, each block $10 \mathrm{~s}$ long, for a total of 24 blocks per scan. In Experiment 2 an additional localizer run was included to identify the horizontal and vertical meridians. Wedges of $100 \%$ contrast polar checkerboards phase-reversing at $8 \mathrm{~Hz}$ were presented along either the horizontal or vertical meridian of the visual field in alternating blocks, each block $10 \mathrm{~s}$ long, for a total of 16 blocks per scan.

Throughout all blocks in all functional scans, participants were engaged in a conjunctive color-shape detection task in the center of the screen, to encourage fixation and maintain engagement and wakefulness. Participants were instructed to press a button when a green circle appeared in a series of small, briefly presented color shapes (green circle, green square, blue square, blue hexagon, red hexagon, red star, purple star, or purple circle), superimposed on the experimental stimuli (i.e., gratings/checkerboards). Shapes subtended $0.5^{\circ}$ of visual angle, and appeared for $66 \mathrm{~ms}$ in a rate of 1.33/s. Performance in this task was of no interest for the main experimental design, but served as a measure of compliance; runs in which the hit rate was $<60 \%$ were excluded from the fMRI analysis, as detailed below. We further examined the possible contribution of performance differences to our neuroimaging findings by directly comparing the hit rate between groups. This analysis showed an overall high hit rate, and no differences between ASD and NT $\left(t_{(46)}=\right.$ $-0.49, p=0.628$; Fig. $7 a$ ).

MRI data processing. Data were preprocessed using BrainVoyager QX v2.8.4 (Brain Innovation) software. EPI data were motion-corrected, corrected for distortion due to magnetic field inhomogeneities, highpass filtered (cutoff $=2$ cycles/scan), and coregistered to the AC-PCaligned $\mathrm{T} 1$ structural scan.

ROIs were identified from the localizer scan data using correlational analyses with an initial threshold of $p<0.05$ (Bonferroni corrected). In Experiment 1 ROIs were defined for each hemisphere in two anatomical regions: a region of early visual cortex in the occipital pole right outside the calcarine sulcus, selective to the retinotopic position of the central stimulus (referred to here as V1; the activation to a central $2^{\circ}$ stimuli lies within the foveal confluence, where V1/2/3 could not be distinguished with current resolution; Schira et al., 2009); and motion-selective MT+ in the lateral occipital lobe (Fig. 1b). In Experiment 2 seven ROIs were defined for each hemisphere: regions of visual cortex in the occipital pole corresponding to dorsal and ventral V1, V2, and V3, selective to the retinotopic positions of the peripheral stimuli in the four quadrants of the visual field, and motion-selective MT + . ROI position was defined manually, verified by visualization on an inflated model of the cortical white matter surface (Figs. 2b, 5b). Peripheral V1, V2, and V3 ROIs were segregated using the meridian localizer scan results as a guideline. The top 20 most significant voxels (in functional space) within each ROI were used in the analysis. In a few cases, where there were not 20 functional voxels within an ROI that met the statistical threshold above, the threshold was relaxed until 20 voxels from the surrounding region were in- cluded, resulting in a constant ROI size of 20 voxels for all regions and all participants. In Experiment 2 there were some cases where no obvious cluster of voxels was present after the threshold was lowered, or where borderlines between regions were not clear, and these ROIs were excluded. This resulted in one ventral V2 ROI and two ventral V3 ROIs excluded for NT participants, no such exclusions occurred among ASD.

Average time courses across the 20 voxels in each ROI were determined for each block. Percentage-transformed time courses were then calculated for each block: first, for each stimulus block, we extracted 12 time points corresponding to $-4 \mathrm{~s}$ before stimulus onset to $18 \mathrm{~s}$ after stimulus onset. Then we converted the values to percentage signal change relative to the mean value of time points $-4,-2$, and 0 (reflecting the best-estimate of "baseline" before stimulus onset). Specifically, each time course was normalized by subtracting and dividing by the mean of the prestimulus time points and multiplying by 100 . The resulting block time courses were then averaged over blocks of the same condition, across hemispheres, and across runs and sessions, yielding one such time course for low contrast and another for high contrast for each subject. Finally, to extract a univariate measure to be used in the statistical analyses, the average response magnitude in time points $8-12 \mathrm{~s}$ was calculated from each of these time courses. Some blocks/runs were excluded from averaging because of head motion or low task performance, according to the following criteria: data for a given block was excluded because of head motion if the framewise displacement in successive TRs was $>0.9$ mm (Power et al., 2014; Siegel et al., 2014), up to and including 8 TRs before the stimulus block or 2 TRs after it. If more than one-half the blocks of either condition were excluded, or a subject had a hit rate of $<60 \%$ in the central fixation task, the whole run was excluded. If $<2$ runs remained for a subject, than the subject was excluded from analysis completely. This procedure resulted in 6-24 blocks per condition for each participant included in the final data analysis [ASD participants: mean $21.58(\mathrm{SD}=4.19)$; NT participants: mean $22.38(\mathrm{SD}=3.64) ; t_{(46)}=$ $-0.68, p=0.497]$.

A secondary analysis was conducted to further control for head movements and other non-neural noise by projecting out signal associated with noise based on the compCor procedure (Behzadi et al., 2007) as implemented in FMRIPREP v1.5.2 (Esteban et al., 2019). Principal components were estimated for the two CompCor variants: temporal (tCompCor) and anatomical (aCompCor). A mask to exclude signal with cortical origin was obtained by eroding the brain mask, ensuring it only contained subcortical structures. tCompCor components were then calculated including only the top 5\% variable voxels within that subcortical mask. For aCompCor, components were calculated within the intersection of the subcortical mask and the union of CSF and WM masks calculated in T1w space, after their projection to the native space of each functional run. Up to 6 components from each variant, or less if the cumulative explained variance exceeded $50 \%$, were included in addition to the six motion parameters as regressors in a GLM, and the residual time courses were used for consecutive processing as in the main analysis. The results of this analysis yielded qualitatively identical results to the main analysis, and hence are not further reported.

Head motion analysis. A head motion censoring procedure was applied before event related averaging as described in the MRI data processing section, and an additional noise removal step using compCor was shown to yield identical results. However, these preprocessing steps do not preclude residual head motion in the signal. Although the opposite direction of effects found in V1 and MT+ (attenuation in V1 and amplification in MT+ among the ASD group; see Results) argues against the possibility that these findings are driven by differences in data quality between the groups, we further examined the possible effect of head motion on the results. Unsurprisingly, the average framewise displacement (FD) in the data segments that were included in the final analysis was higher in ASD than in NT $\left(t_{(46)}=-2.24, p=0.030\right.$; Fig. $\left.7 b\right)$. However, when splitting the ASD group according to the median value of V1 response amplitude to high contrast stimuli, comparing participants with high response to participants with attenuated response, there were no differences in $\mathrm{FD}\left(t_{(22)}=-0.010, p=0.923\right.$; Fig. $\left.7 b\right)$. The same pattern of results was obtained when splitting the group according to V1 response amplitude to low contrast stimuli or according to MT+ response ampli- 
a

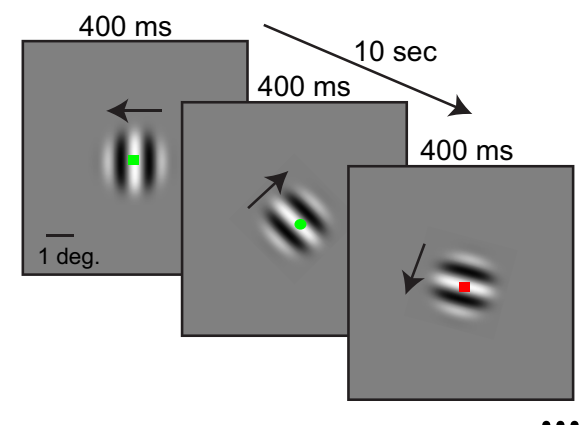

C

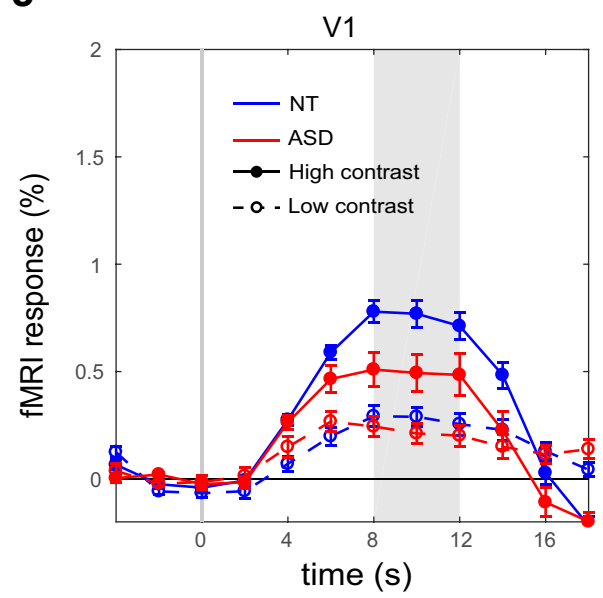

e

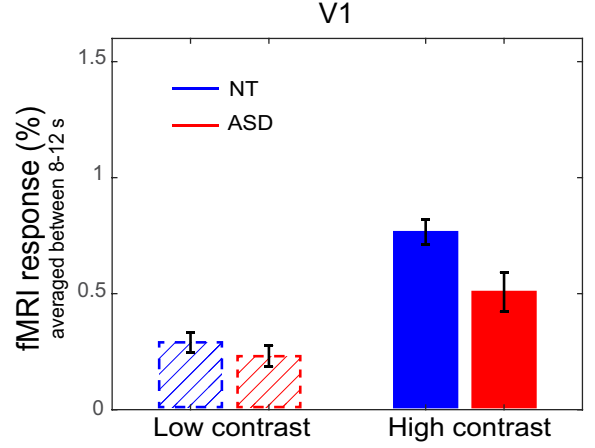

b

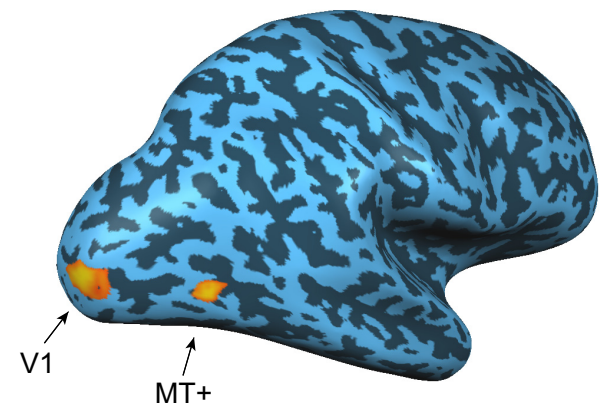

d

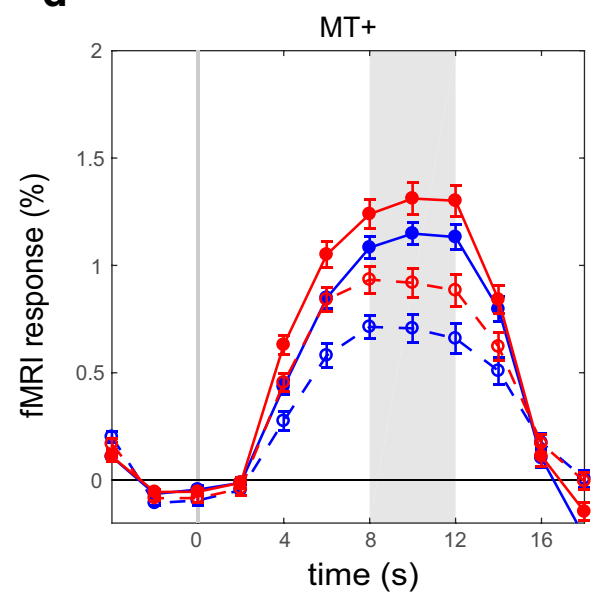

f

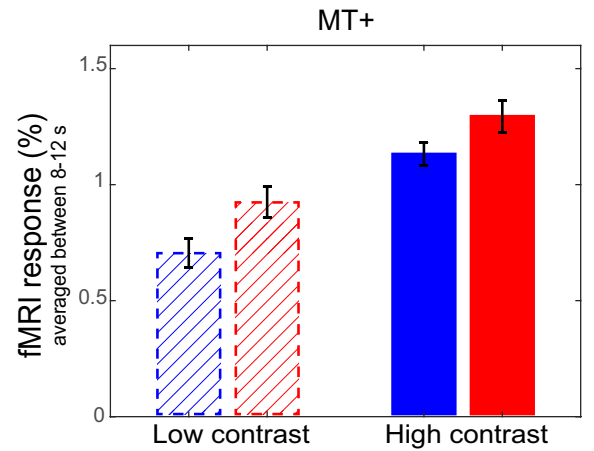

Figure 1. Task, ROls, and fMRI responses. $\boldsymbol{a}$, Drifting gratings ( $2^{\circ}$ diameter) were presented in the center of the screen, changing direction every $400 \mathrm{~ms}$. Stimuli were high contrast ( $98 \%$ ) or low contrast (3\%) in different blocks. Participants were asked to press a button when a green circle appeared. Blocks were 10 s long and alternated with 10 s blocks of the fixation task only. $\boldsymbol{b}$, ROls in left and right $\mathrm{V} 1$ and MT + were defined using independent localizer scans. R0Is in the right hemisphere are shown for an example NT participant overlaid on an inflated cortical surface. (c, $\boldsymbol{d}$ ) time courses and $(\boldsymbol{e}, \boldsymbol{f})$ average magnitude of peak fMRI responses of NT (blue) and ASD (red) participants, for high contrast (solid lines) and low contrast (broken lines) stimuli. Shaded regions in c-d deonte the time window for averaging in e-f. Error bars denote standard error of the mean (S.E.M). ASD responses are higher than NT responses in MT+ but lower in V1.

tude to either contrast level. Furthermore, average FD was not correlated with $\mathrm{V} 1$ response amplitude (high contrast: $r_{22}=0.12, p=0.394$; low contrast: $\left.r_{22}=-0.26, p=0.073\right)$ or with $\mathrm{MT}+$ response amplitude (high contrast: $r_{22}=-0.15, p=0.307$; low contrast: $r_{22}=-0.16, p=$ 0.266). To summarize, although ASD participants had more head motion during the scans than NT, this difference is not driving the observed group differences in the study.

Eye tracking. When possible, subjects' eyes were tracked during scanning using an EyeLink 1000 Plus eye-tracker, sampling at $1000 \mathrm{~Hz}$. Because of the challenges of eye-tracking in the scanner, especially with participants wearing glasses, we were able to collect good quality data for $56 \%$ of subjects ( 11 ASD participants, 16 NT participants). We identified fixation periods using a dispersion-based fixation detection algorithm (Anliker, 1976; Blignaut, 2009) along with a subtractive post hoc drift correction (Vadillo et al., 2015). Fixations were defined within set peri- ods of at least $100 \mathrm{~ms}$ for which the maximum distance of any gaze position measurement within the set compared with the set's centroid does not exceed a threshold radius of $1^{\circ}$. Next, post hoc drift correction was performed by calculating the average gaze position across all fixations within a $10 \mathrm{~s}$ period, taking the difference between this average fixation position and the intended fixation position (i.e., the fixation mark at the center of the screen), and subtracting this value from all gaze position measures within the $10 \mathrm{~s}$ period. We found no group differences in eye movement behavior in the subjects whose eyes were successfully tracked: no difference between ASD and NT in the proportion of time spent fixating (two-tailed $t_{(25)}=-0.58, p=0.566$; Fig. $7 c$ ), or in the mean distance of eye position from the fixation mark (two-tailed $t_{(25)}=$ $-1.22, p=0.235$; Fig. $7 c$ ). Notably, one ASD participant had an extreme value in the latter measure; excluding him from the sample did not alter any of the effects reported in the main text. We further compared the 

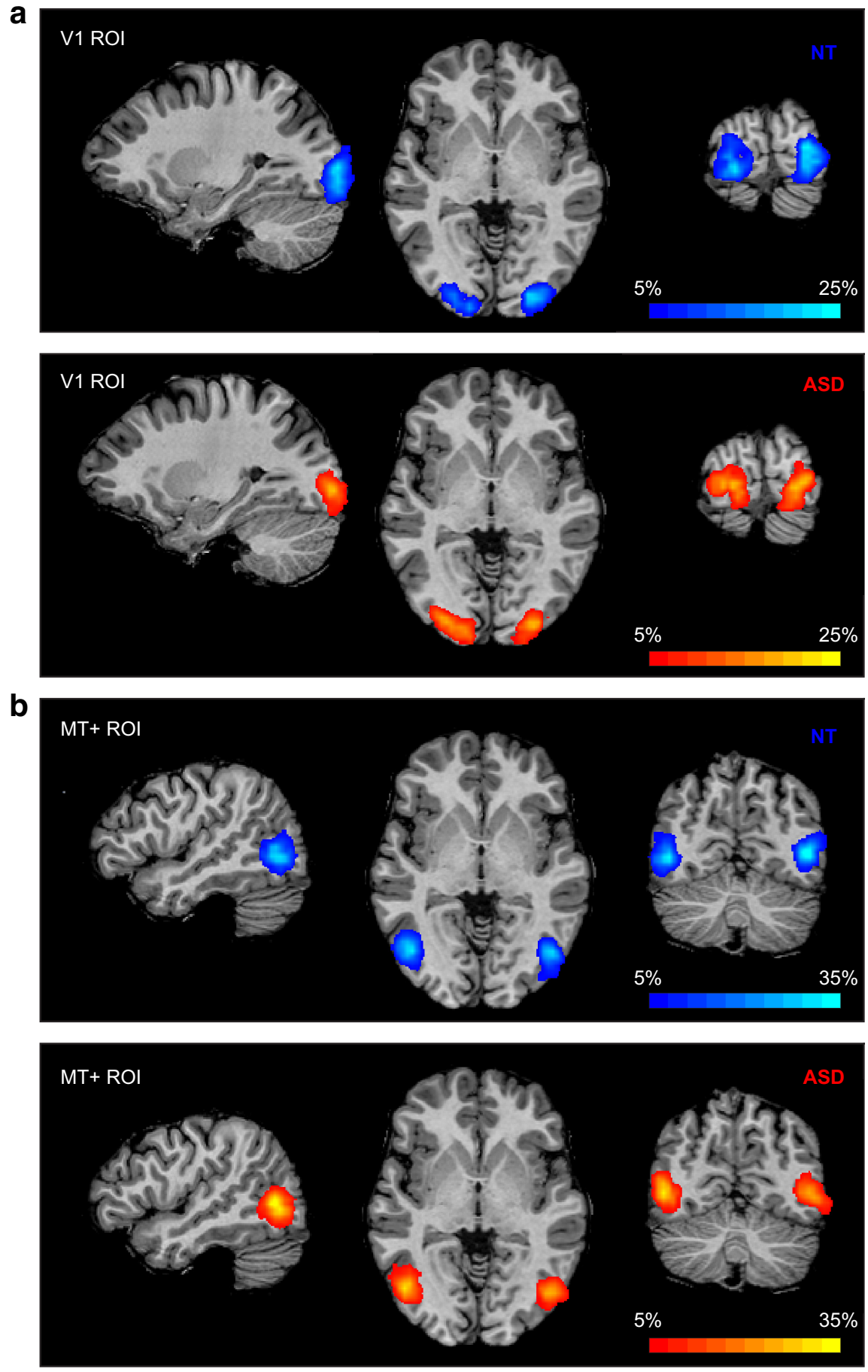

Figure 2. Probability maps showing V1 (a) MT + (b) ROI locations for NT (blue) and ASD (red) participants. Percentage overlap across subjects calculated in Talairach space and displayed on an individual subject's anatomical image.

ASD subjects who had available eye-tracking data to those who did not, to examine the possibility that the eye-tracking data are not representative of our sample, or that eye-tracking data availability is otherwise confounded with our findings. We found that subgroups of ASD participants with $(n=11)$ or without $(n=13)$ eye-tracking data did not differ in any demographic variables (age, sex, or IQ), ASD symptom severity (as assessed with the ADOS-2 total comparison score), or fMRI responses in any of the examined ROIs.

Psychophysical tasks. Stimuli were presented using a ViewSonic PF790 CRT monitor $(120 \mathrm{~Hz})$ with an associated Bits\# stimulus processor (Cambridge Research Systems). The monitor luminance was linearized using custom software. Stimuli were presented on a Windows PC in MAT-
LAB (MathWorks) using Psychtoolbox-3, with a chin rest used to stabilize head position at a viewing distance of $66 \mathrm{~cm}$.

The contrast detection task was described in detail previously (Murray et al., 2018). Briefly, the task was to detect whether a Gabor (sinusoidal luminance modulation within a Gaussian window, $\mathrm{SD}=0.42^{\circ}, \mathrm{FWHM}=1^{\circ}, 1.5$ cycles $/{ }^{\circ}$, vertical or horizontal orientation) was presented at the center of a mean gray background during either the first or second of two possible stimulus presentation intervals. Stimulus contrast was adjusted using a staircase procedure, to determine the lowest contrast that could be detected with $80 \%$ accuracy (i.e., the threshold).

The motion discrimination task is described and reported in detail previously (Murray et al., 2018; Schallmo et al., 2018, 2019b). Briefly, drifting sinusoidal luminance modulated gratings at two different Michelson contrast levels (low $=3 \%$, high $=98 \%)$ and three different sizes (diameter $=0.84^{\circ}, 1.7^{\circ}$, and $10^{\circ}$ ) were presented in the center of the screen on a mean luminance background. Motion speed was 4 cycles/s, and spatial frequency was 1.2 cycles $/{ }^{\circ}$. Gratings were presented within a circular aperture, whose edges were blurred with a Gaussian envelope $\left(\mathrm{SD}=0.21^{\circ}\right)$. Participants were instructed to indicate the motion direction of the grating (left or right). Stimulus duration was adjusted using a staircase procedure to determine the amount of time needed to correctly discriminate motion direction with $80 \%$ accuracy (i.e., the threshold). For the purpose of the current analysis, we computed a geometric mean across thresholds from different conditions, to create an overall estimate of motion sensitivity. For four NT participants and two ASD participants, thresholds were not obtained for the smallest stimulus size; hence, their data from this task were not included. One NT and one ASD participant were excluded from the analysis due to catch trials accuracy $<80 \%$. One additional ASD participant was excluded because his threshold was $>3 \mathrm{SD}$ away from the groups' mean. This resulted in a final sample of 19 NT and 20 ASD participants for the motion discrimination task.

Statistics. Statistical analyses were performed in MATLAB R2017a and in SPSS v19. Experimental task fMRI data were analyzed using three-way mixed repeated-measures ANOVA, with stimulus contrast and ROI as withinsubjects factors and group as a between-subject factor; and group differences in localizer fMRI data and in psychophysical tasks were assessed using two-sample two-tailed $t$ tests. Correlations were assessed using Pearson's correlation coefficient, and correlation coefficients for the two groups were compared using Fisher's $Z$ transform and test.

Data Availability. All data reported in the manuscript, including neuroimaging and behavioral data, is available through the NIH National Database for Autism Research (NDAR). Raw data for experiment 1 is also available on openneuro.org (ds002522).

\section{Results}

We measured sensory driven fMRI responses in early visual cortex in a sample of adult individuals diagnosed with ASD and in a 
a

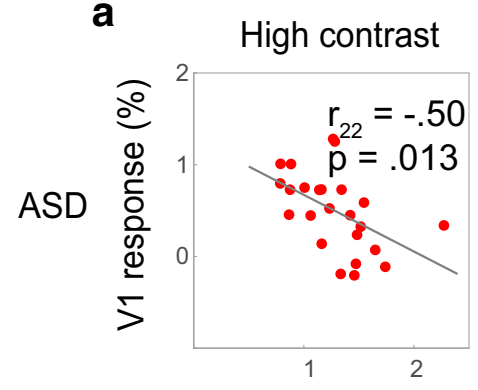

C

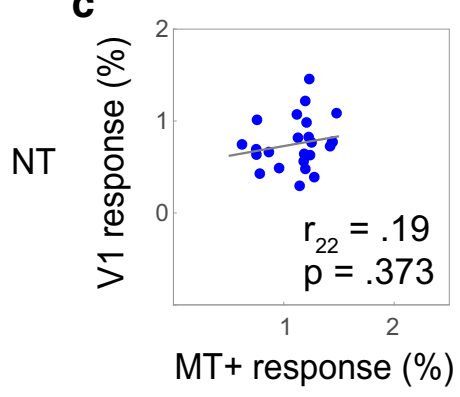

b

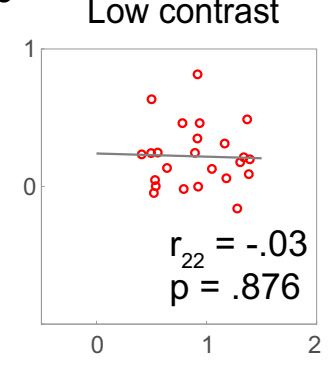

d

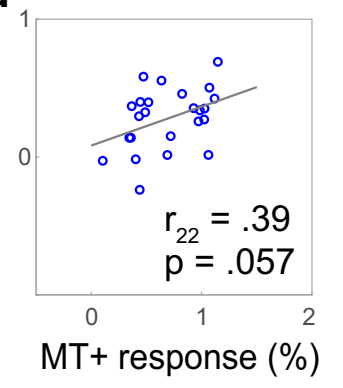

Figure 3. $\quad \mathrm{V} 1$ and $\mathrm{MT}+$ response correlations. Negative correlations between response magnitude in V1 and MT+ among the ASD group $(\boldsymbol{a}, \boldsymbol{b})$, i.e., participants with high MT+ responses have low V1 responses. The opposite is true for NT $(\boldsymbol{c}, \boldsymbol{d})$, where V1 and MT+ magnitudes are positively correlated.

demographically-matched sample of NT control participants. Experimental stimuli were small $\left(2^{\circ}\right.$ diameter $)$ moving sine wave gratings (Fig. 1a) presented at the center of the visual field in blocks of very low contrast (3\%) and very high contrast (98\%). Response amplitudes were measured in two ROIs: an early visual area was at the foveal confluence near the occipital pole, and we refer to it hereafter as V1. The second ROI was human MT complex $(\mathrm{MT}+)$, a higher-order area in extrastriate cortex that responds selectively to visual motion (Figs. 1b,2). To emphasize sensory driven responses and promote stable eye fixation and attentional engagement, participants performed a conjunctive color-shape detection task at the center of the screen throughout all conditions in all scans. fMRI responses were calculated for both low- and high-contrast stimuli relative to a blank (fixation only) baseline condition.

Average time courses (Fig. $1 c-f$ ) showed responses that were strongly modulated by stimulus contrast in both ROIs, as expected (main effect of contrast: $F_{(1,46)}=110.17, p<0.001, \eta_{\mathrm{p}}^{2}=$ 0.71 ). Responses in MT+ were overall higher than in V1 (main effect of ROI: $F_{(1,46)}=90.37, p<0.001, \eta_{\mathrm{p}}^{2}=0.66$ ). Importantly, there was a significant interaction of ROI and group $\left(F_{(1,46)}=\right.$ $\left.8.62, p=0.005, \eta_{p}^{2}=0.16\right)$. Follow-up simple effects analyses, collapsing across contrasts, revealed stronger MT + responses in ASD than in NT, in line with the E/I model prediction $t_{(46)}=$ $2.22, p=0.032$ ). However, $\mathrm{V} 1$ responses had the opposite pattern, with responses being lower in ASD than in NT $t_{(46)}=$ $-2.41, p=0.020)$. No other effects in the ANOVA were significant.

Moreover, the most striking difference between the groups was in the relationship of responses in V1 and MT + across individual participants (Fig. 3). There was a significant negative correlation between $\mathrm{V} 1$ and $\mathrm{MT}+$ response magnitudes to high contrast stimuli among individuals with ASD: participants with lower $\mathrm{V} 1$ responses showed higher $\mathrm{MT}+$ responses $\left(r_{22}=-0.50\right.$, $p=0.013)$. This relationship between $\mathrm{V} 1$ and $\mathrm{MT}+$ was not

evident in the NT individuals, and even had the opposite trend $\left(r_{22}=0.19, p=0.373\right.$; significantly different from the correlation among ASD as tested with Fisher's $z$ test: $z=2.41, p=0.016$ ).

Importantly, attenuation of V1 seems to be stimuli-specific. When examining the responses to a flickering checkerboard during the localizer scan, all participants had robust and comparable responses to the stimuli (Fig. 4a). Consequently, V1 was reliably defined for all participants. There were no group differences in the size of the ROI (fixed to 20 voxels; see Materials and Methods) or in its spatial location (Fig. 2, heat maps). At the same time, $\mathrm{MT}+$ was not as strongly driven by the checkerboard stimuli as it was by drifting gratings, and these responses were not different between groups $\left(t_{(46)}=0.79, p=0.435\right.$; Fig. $\left.4 b\right)$. This pattern of results suggests that the attenuated V1 responses are stimulusspecific, and depend on the motion information and/or on the activation of $\mathrm{MT}+\mathrm{V} 1$ attenuation is observed when the stimuli strongly activate $\mathrm{MT}+$, but not in the flickering checkerboard condition when MT+ is only weakly stimulated.

An inspection of individual subjects' data revealed that for some ASD participants, responses to the drifting gratings in the experimental runs were not only attenuated but were even below the baseline response, suggesting a suppressive effect. Moreover, in these participants with the most attenuation, there was a reversal in contrast response, with responses for high contrast stimuli being lower than responses to low contrast stimuli (Figs. $4 d$, example participants, 5, all individual data). Based on these observations, we suggest that enhanced suppressive feedback from higher-order area MT+ is underlying attenuated V1 responses.

To further explore the notion that suppressive feedback from $\mathrm{MT}+$ drives the observed attenuation of $\mathrm{V} 1$ responses, we next examined V1 responses to an independent set of stimuli. If the attenuation of V1 is a stable individual-difference trait, we would predict it would manifest in responses to a variety of moving stimuli. Thus, we examined V1 response in the MT-localizer scan; a scan during which we know that MT+ is strongly engaged. In this scan, blocks of drifting medium contrast (15\%) gratings alternated with blocks of stationary gratings. The responses in V1 to this contrast were overall low, because of the constant visual stimulation; however, response magnitudes of ASD participants were correlated with response magnitudes in the main experiment $\left(r_{22}=0.42, p=0.041\right.$; Fig. $\left.4 c\right)$, indicating stability of V1 attenuation across scans.

Interestingly, response magnitudes in both $\mathrm{V} 1$ and $\mathrm{MT}+$ were differentially correlated with autism symptom severity, as quantified using the total comparison score from the ADOS-2 (Fig. 6; $\left.\mathrm{V} 1: r_{22}=-0.46, p=0.024 ; \mathrm{MT}+: r_{22}=0.41, p=0.047\right)$, indicating that response magnitude in visual cortex in ASD may be clinically relevant.

We ruled out the contribution of a number of potential confounding variables to the attenuated V1 response, including differences in fixation task performance, head motion, and eye movement patterns (Fig. 7). We also rescanned a subgroup of ASD participants on a different session, to test for reliability of the response amplitude effects, and demonstrated a highly replicable pattern of $\mathrm{V} 1$ and $\mathrm{MT}+$ responses (Fig. 8).

In the experiment described thus far, stimuli were presented in the center of the visual field, hence the analysis was confined to a region in the foveal confluence, likely within V1. To further investigate response magnitude in other regions along the visual pathway, specifically intermediate-order regions V2 and V3 lying in between V1 and MT+, we conducted a second experiment with stimuli presented in the periphery of the visual field. Drifting gratings subtending $3^{\circ}$ of visual angle were presented in the four 
a

V1 localizer:

flickering checkerboard stimuli

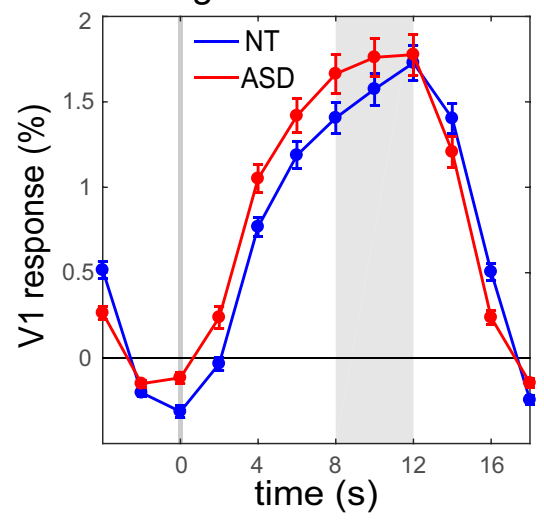

b
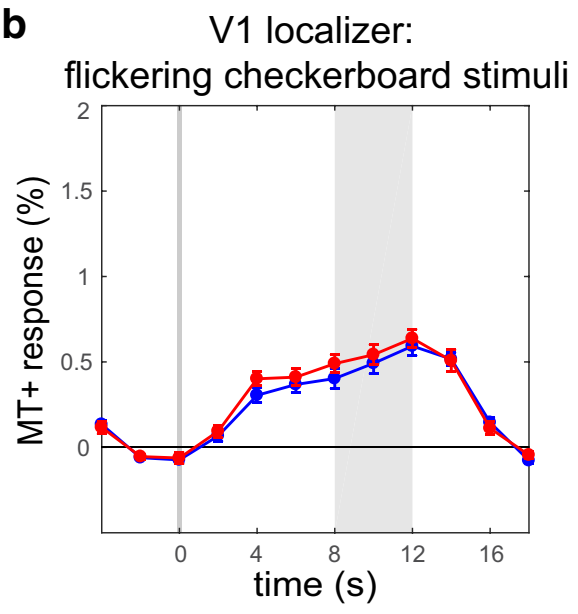

C

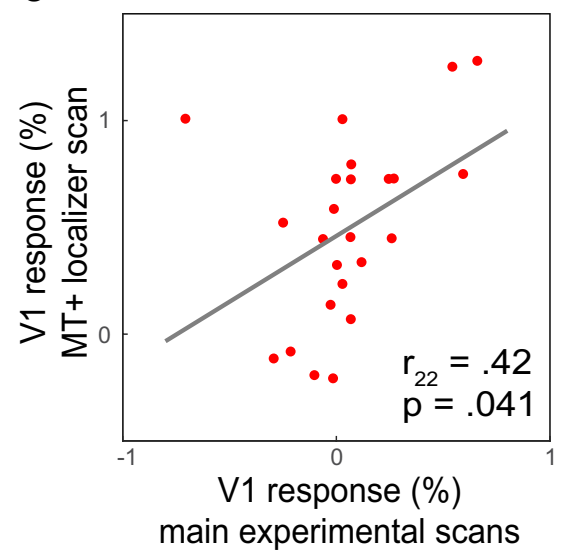

d

\section{V1 localizer Experimental scans: drifting gratings}
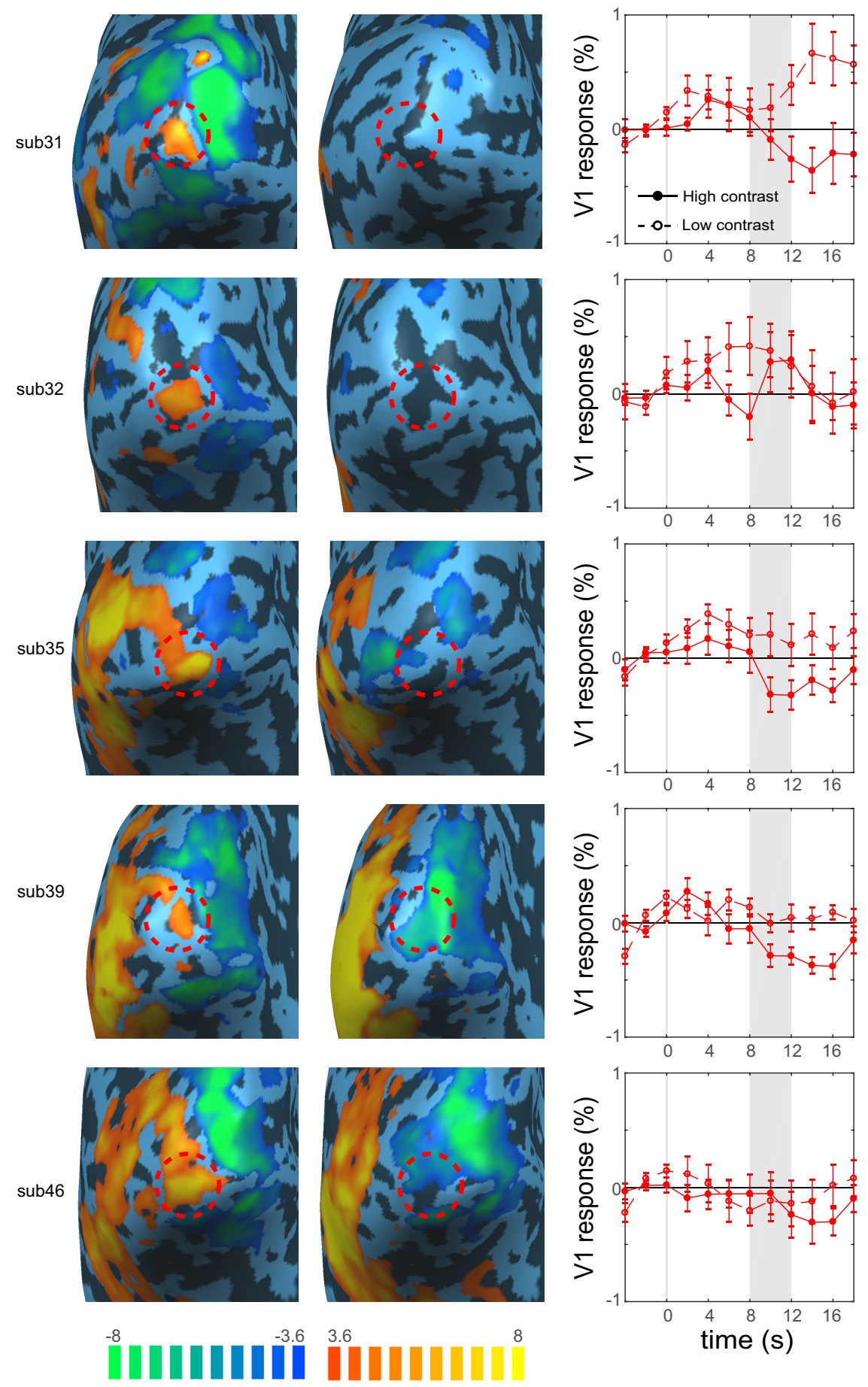

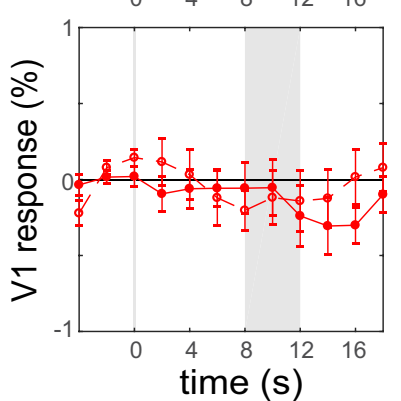

Figure 4. FMRI responses to the localizer stimuli, and drifting grating responses in individuals with strong V1 attenuation. $\boldsymbol{a}$, ASD fMRI responses to flickering checkerboard stimuli are robust and similar to the responses in NT. $\boldsymbol{b}$, MT + responses to flickering checkerboard stimuli are low and similar between groups. $\boldsymbol{c}$, V1 responses during the MT + localizer scan, where MT + is strongly activated, are correlated with responses to drifting gratings during the main experimental scans, demonstrating that individuals with attenuated responses show this pattern across scans. $\boldsymbol{d}$, Individual participants with the most V1 attenuation. Early visual cortex responses to flickering checkerboard stimuli during the localizer scan (first column) with V1 R0I denoted with a red dashed circle. The responses in the same regions to high contrast drifting gratings during the experimental scans (second column) were low or even below baseline. For some, responses to high contrast stimuli were lower than responses to low contrast stimuli (third column). The values in the brain maps represent $t$ values from a GLM analysis. Shaded regions in the timecourses denote time window for averaging the peak magnitude of fMRI response. Error bars denote S.E.M. 

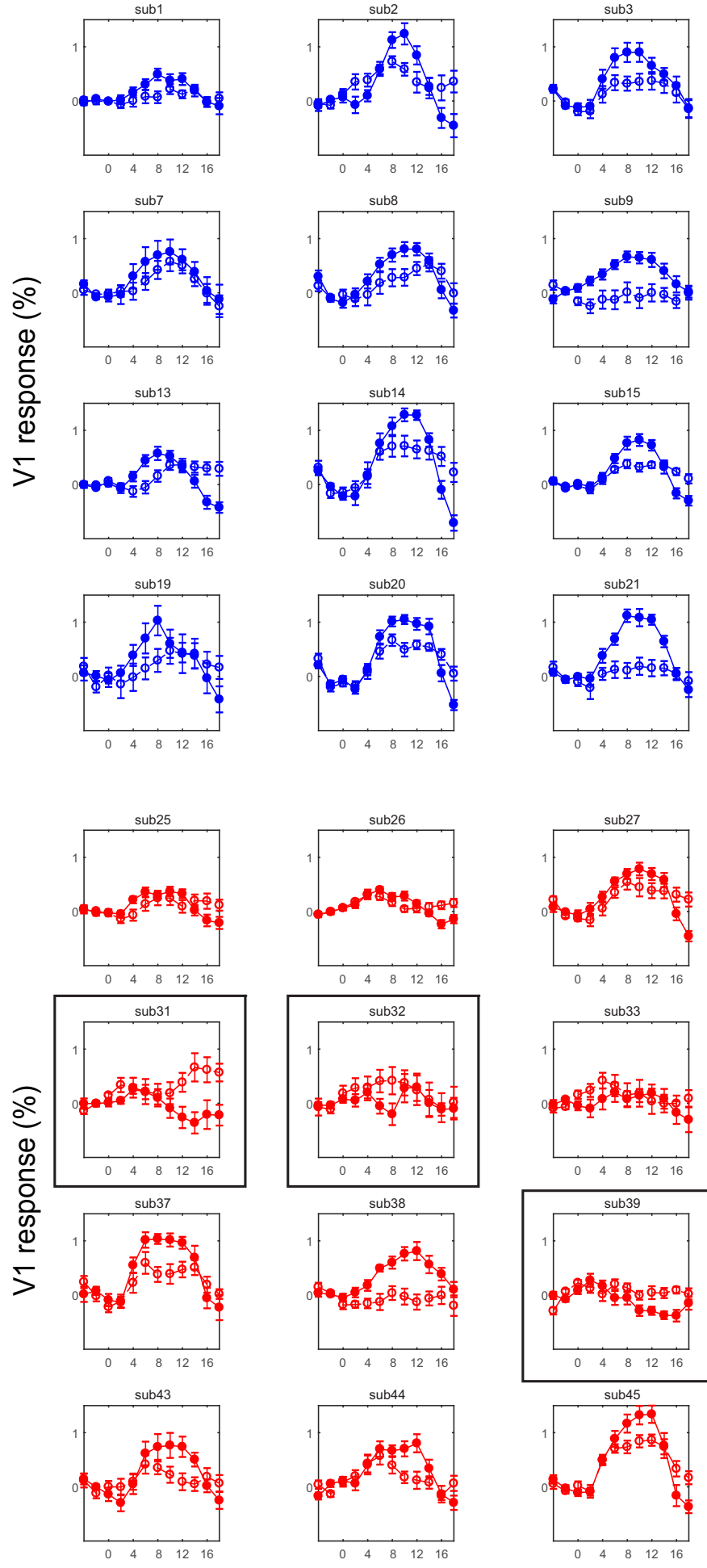

time (s)
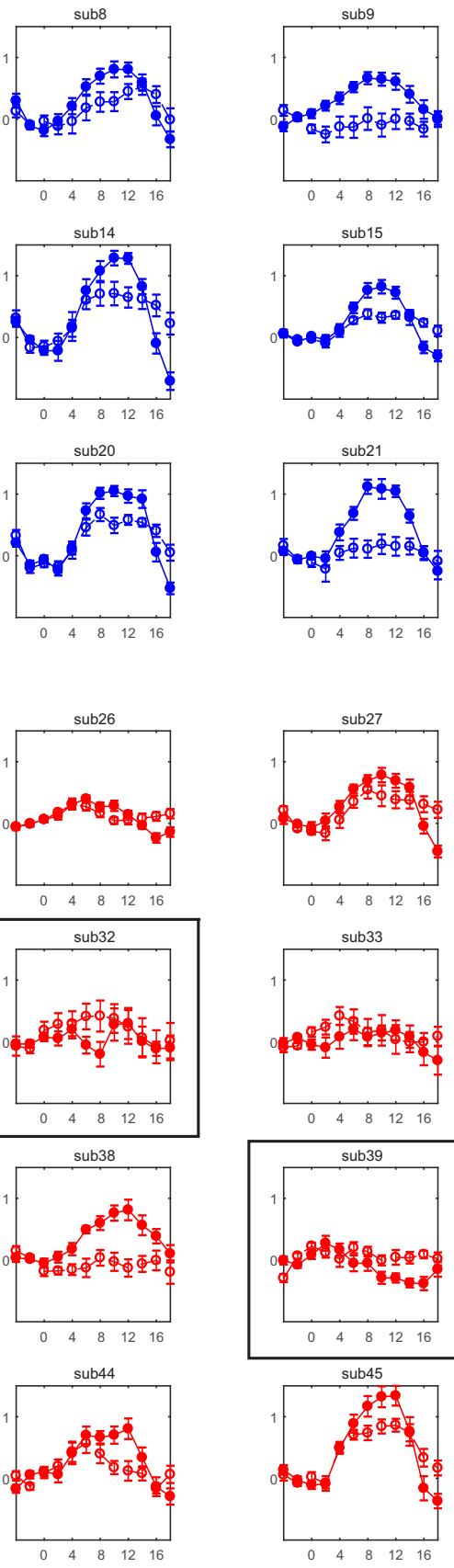

time (s)


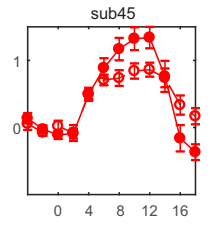

time (s)

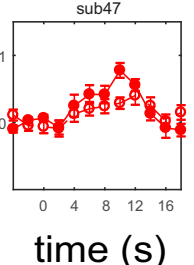

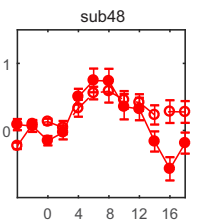

time (s)

Figure 5. V1 responses to drifting gratings stimuli in individual participants. Responses to high contrast gratings (filled circles, solid lines) are typically higher than responses to low contrast gratings (empty circles, broken lines). However for a few ASD participants, this patterns is reversed (indicated with a black frame; also depicted in Fig. $4 d$ ). Error bars denote S.E.M.

quadrants of the visual field simultaneously, at an eccentricity of $5^{\circ}$, with all other parameters identical to the primary experiment (Fig. 9a). ROIs were defined in ventral and dorsal V1, V2, and V3, and in area MT+ (Fig. 9b; see Materials and Methods). Re- sponses were strongly modulated by stimulus contrast in all ROIs and for both the ASD and NT groups, as expected (all $F_{(1,14)}>$ $46.7, p$ values $\left.<0.001, \eta_{\mathrm{p}}^{2}>0.77\right)$. Response amplitudes in the ASD group were slightly higher than in the NT group in all 
a

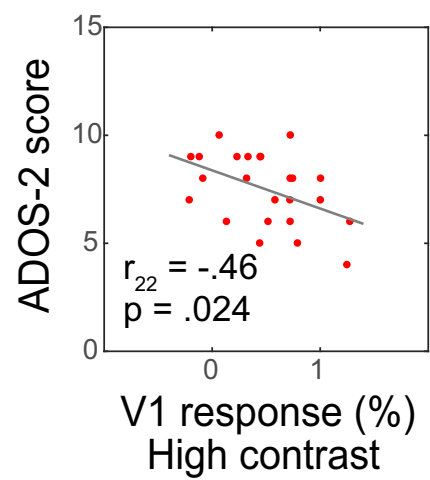

b

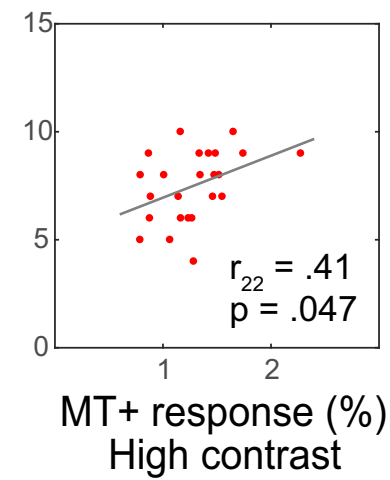

Figure 6. FMRI response amplitudes correlate with ASD symptom severity. V1 (a) and MT+ (b) responses are significantly correlated with the ADOS-2 total comparison score, i.e., participants with high MT+ and Low V1 responses experience more severe behavioral symptoms of ASD.

a

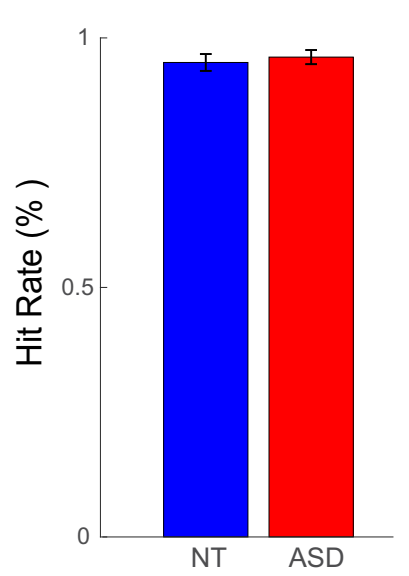

C

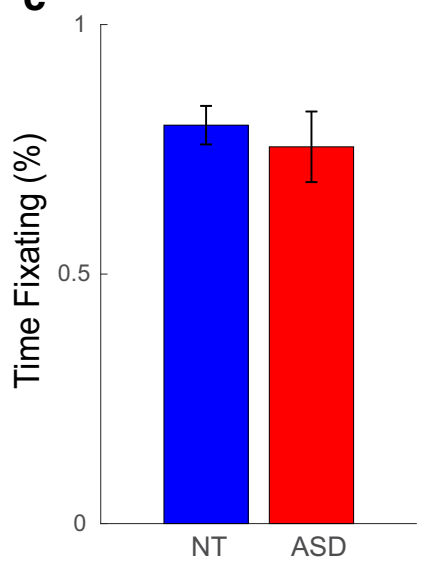

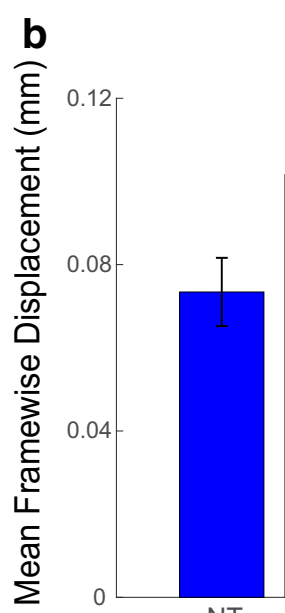

NT
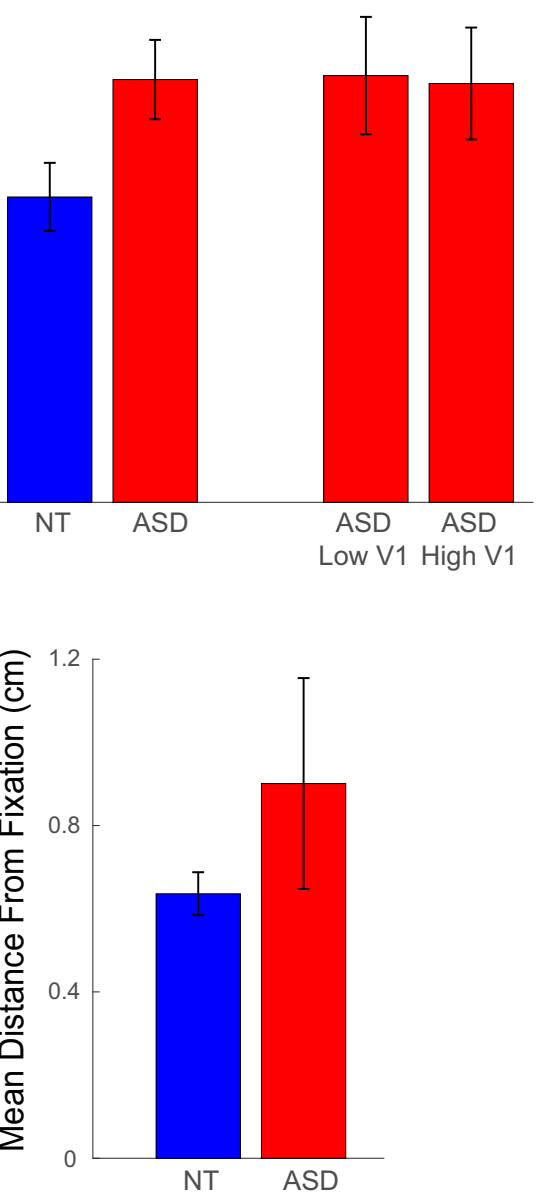

Figure 7. Examination of possible data quality confounds. $\boldsymbol{a}$, No group differences in performance of the conjunctive shapecolor detection task at fixation during fMRI scans. $\boldsymbol{b}$, More head motion in ASD than in NT. However, no differences in head motion between ASD participants with low V1 responses and those with high V1 responses. $c$, No group differences in adherence to fixation instructions during fMRI scans. See detailed description of these analyses in Materials and Methods. Error bars denote S.E.M.

cally mapped to the center of the visual field (i.e., the perifoveal region).

Finally, to examine possible behavioral consequences of differences in neural response magnitude in visual cortex, we measured performance on two psychophysical tasks using foveal stimuli: contrast detection, associated with early visual cortex activity (Boynton et al., 1999; Ress and Heeger, 2003); and motion perception, known to be tightly linked to MT+ responses (Turkozer et al., 2016; Chen et al., 2017). Contrast detection thresholds were not different between ASD and NT $\left(t_{(46)}=-0.05, p=0.957\right.$; Fig. 10a), whereas motion discrimination thresholds were significantly lower in ASD than in NT $\left(t_{(37)}=2.77, p=0.009\right.$; Fig. $\left.6 b\right)$, indicating typical contrast sensitivity but higher motion sensitivity in ASD. These psychophysical thresholds did not correlate with the fMRI measurements.

\section{Discussion}

Our results demonstrate differences in fMRI response magnitude in participants with ASD that vary between adjacent stages of processing in the visual pathway: attenuated responses in $\mathrm{V} 1$ and increased responses in higher-order area MT+. This pattern of magnitude change is stimulusspecific; V1 responses were attenuated for moving stimuli (drifting gratings) but not for static stimuli (flickering checkerboards). Moreover, the attenuation of $\mathrm{V} 1$ responses was proportional to the responses in the motion-sensitive area $\mathrm{MT}+$, suggesting a causal link may exist between the two.

Notably, differences in attention to or away from the visual stimuli could not explain the marked attenuation of $\mathrm{V} 1$ responses in ASD. Participants were performing a conjunctive detection task at fixation during all fMRI scans with equivalently high accuracy in both groups and across different background stimuli (e.g., drifting gratings and flickering checkerboards). If attenuated $\mathrm{V} 1$ responses to the gratings were attributed to filtering or suppression of the gratings because of competition with the fixation task, similar attenuation would have been observed for static stimuli. Furthermore, such attentional effects are expected to occur in MT as well; however, our results point to an opposite pattern of results, increased MT responses concurrent with attenuated V1 responses. Similarly, fixation stability as measured with an eye tracker was equivalent across ASD and NT participants, and compliance with fixation instructions was overall high, ruling out the possibility of eye movements underlying the observed pattern of responses.

The visual system is hierarchically organized, with information traveling for-

ROIs, however, these group differences were not significant $\left(\right.$ all $F_{(1,14)}<2.71, p$ values $>0.162$; Fig. $\left.9 c-f\right)$. Interestingly, this includes V1 responses, indicating that attenuation of early visual response is restricted to subregions of V1 retinotopi- ward from V1 to MT+, both directly and through V2 and V3 (Ungerleider and Desimone, 1986; Felleman and Van Essen, 1991). In addition to these feedforward projections, feedback 


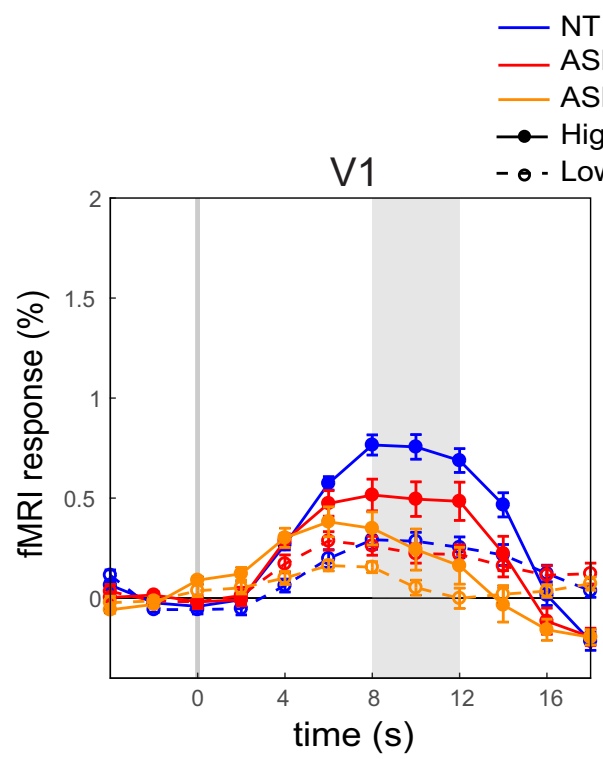

ASD experiment 1

ASD experiment 2

- High contrast

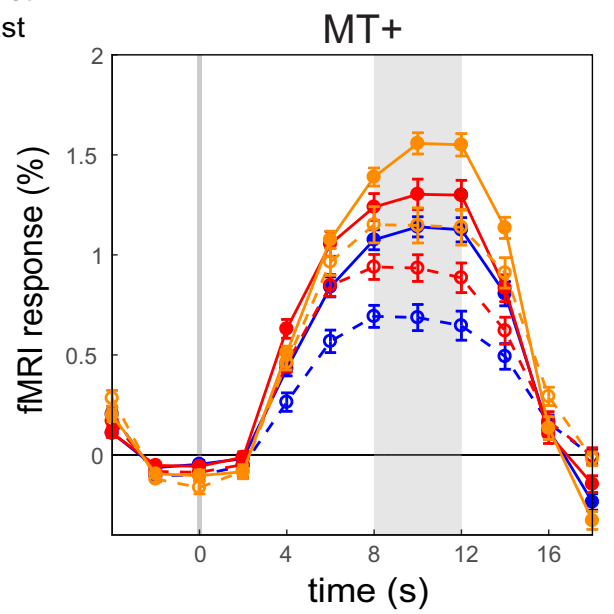

Figure 8. Replication of Low V1 and high MT + responses in ASD. ASD participants who attended Experiment 2 were also rescanned with the original paradigm from Experiment 1, to examine the repeatability of the findings. Attenuation of $\mathrm{V} 1$ and amplification of $\mathrm{MT}+$ were replicated in this second scan. Shaded regions denote time window for averaging the peak magnitude of fMRI response. Error bars denote S.E.M.

a

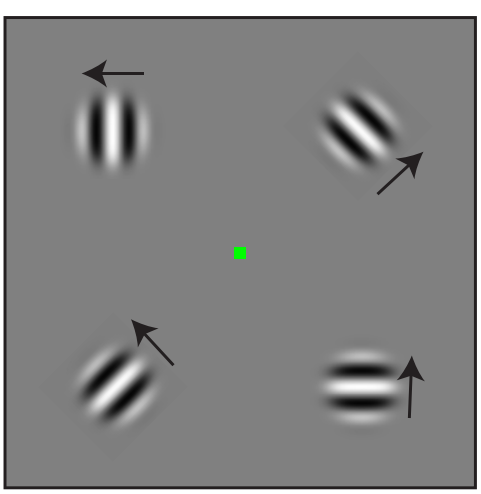

d

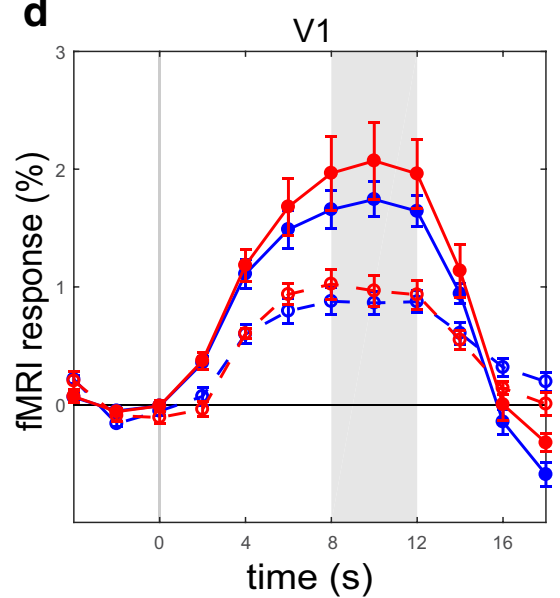

b

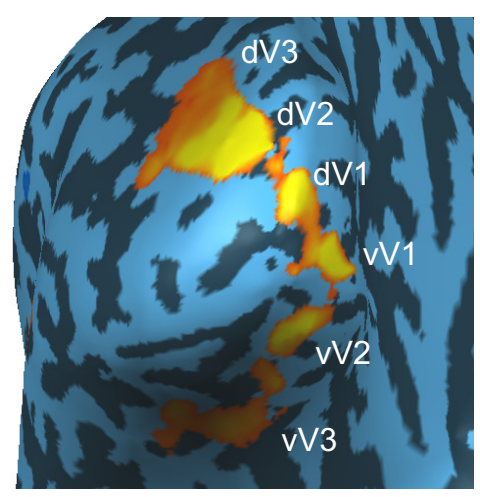

e

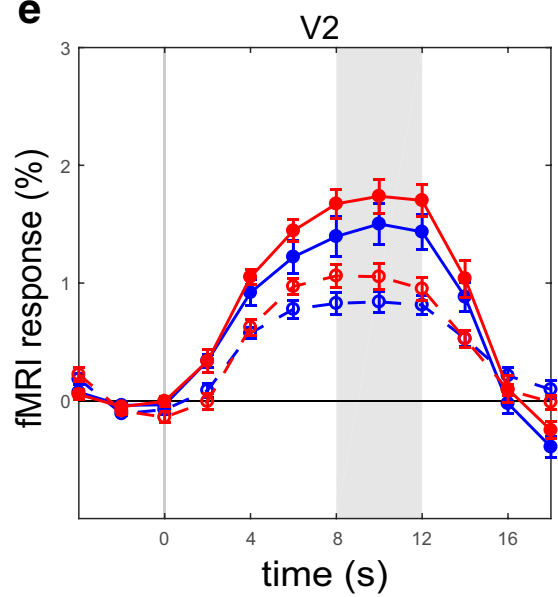

C
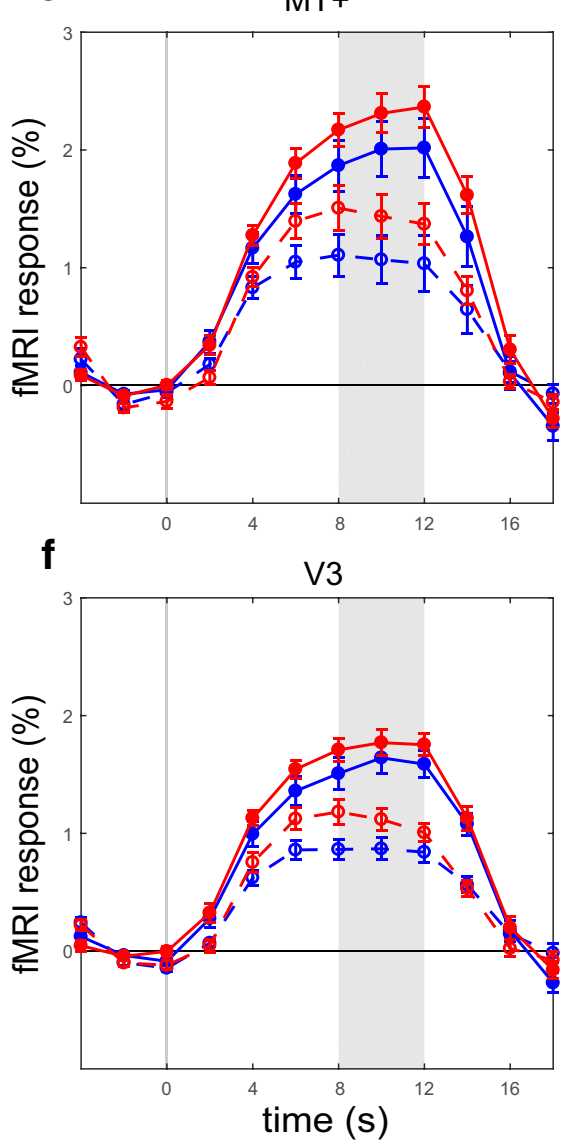

Figure 9. Design and results of Experiment 2: peripheral presentation of gratings stimuli. $\boldsymbol{a}$, Gratings ( $3^{\circ}$ diameter) drifting simultaneously in the four quadrants of the visual field, centered $5^{\circ}$ away from fixation. All other task details identical to Experiment 1.b, ROIs in left and right ventral (v) and dorsal (d) V1, V2, and V3, and MT + were defined using independent localizer scans. ROIs in the left hemisphere are shown for an example ASD participant. $\mathbf{c}-\boldsymbol{f}$, Response magnitude in ASD is similar and somewhat higher than response in NT in all ROIs, including V1. Shaded regions in the timecourses denote time window for averaging the peak magnitude of fMRI response. Error bars denote S.E.M. 
a

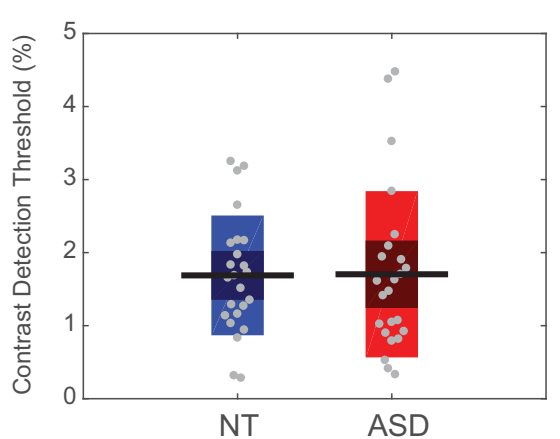

Figure 10. Visual psychophysics performance. $\boldsymbol{a}$, Contrast detection thresholds are similar across ASD and NT participants. $\boldsymbol{b}$, Motion discrimination thresholds are lower in ASD than in NT, indicating higher motion sensitivity. Gray circles represent individual participants, horizontal black lines represent group mean. Dark-shaded areas represent 1 SD from the mean, and the colored bars represent a 95\% confidence interval around the mean.

from MT+ to V1 is also well established (Maunsell and van Essen, 1983; Shipp and Zeki, 1989; Bullier, 2001; Pascual-Leone and Walsh, 2001; Muckli et al., 2005; Silvanto et al., 2005; Sillito et al., 2006) with inhibitory feedback connections outnumbering excitatory feedback connections (Bullier et al., 1996; Hupé et al., 1998). Given this feedforward and feedback circuitry, we considered two possible general mechanisms that could explain the relationship between V1 and MT+ responses in our experiment. First, V1 responses could be intrinsically attenuated via abnormal V1 circuitry in ASD, and the enhancement of MT+ responses may be a consequent of a compensatory response to attenuated V1 input. This possibility is consistent with homeostatic mechanisms that are known to modulate excitation around a set point (Turrigiano, 2011, 2012; Gainey and Feldman, 2017), and hypothesized to be altered in ASD (Ramocki and Zoghbi, 2008; Nelson and Valakh, 2015). An alternative possibility is that the attenuated V1 responses in ASD result from enhanced suppressive feedback from MT+.

There are four observations that lead us to favor a suppressive feedback mechanism. First, attenuation in V1 only occurred for motion stimuli that strongly drive MT + responses. V1 responses for checkerboards stimuli, that do not strongly engage $\mathrm{MT}+$, were typical. Second, in at least a subset of subjects with ASD, attenuation in V1 was stronger and even below baseline for high contrast stimuli. In this subset of subjects this resulted in inverse contrast response functions; higher responses in V1 for low contrast stimuli than high contrast stimuli. It is difficult to explain this observation via a compensatory mechanism. However, enhanced suppressive feedback predicts that any condition, such as an increase in luminance contrast, that increases MT + responses will result in a corresponding decrease in V1 responses. The third important observation is that the attenuated V1 responses were observed only when the stimuli were presented in the center of the visual field, consistent with known suppressive feedback pathways that specifically target foveal retinotopic cortex (Williams et al., 2008; Chambers et al., 2013; Fan et al., 2016). Finally, our psychophysical results demonstrate that contrast detection thresholds were equivalent between ASD and NT individuals. Contrast detection is a task that likely depends heavily on the integrity of feedforward processing in early visual cortex (Boynton et al., 1999; Ress and Heeger, 2003). The fact that no significant differences were observed suggests intact feedforward processing of static visual stimuli in early stages of the visual pathway in ASD, and is consistent with previous reports of typical low-level visual processing (de Jonge et al., 2007; Koh et al., 2010a; Tavassoli et al., 2011). Motion discrimination thresholds, on the other hand, were lower in ASD than in NT, in line with previous findings (Foss-Feig et al., 2013; but for the opposite result, see Schauder et al., 2017; Sysoeva et al., 2017). This result is consistent with the higher fMRI response found in MT+ in the ASD group. It is also consistent with the broader literature of atypical motion perception in ASD (Spencer et al., 2000; Koh et al., 2010b; Chen et al., 2012; Robertson et al., 2012; Manning et al., 2015). Overall, although the evidence appears to favor enhanced suppressive feedback in ASD over a compensatory mechanism, future research that more precisely targets the dynamics of this effect (e.g., disruption of MT+ responses via TMS) is necessary to better understand the underlying processes.

Previous imaging studies examining visual processing in ASD have yielded mixed results. MT+ response has been reported to be low (Herrington et al., 2007), high (Brieber et al., 2010), or typical (Koldewyn et al., 2011) in ASD. Similarly, some studies have found typical responses in V1 (Dinstein et al., 2012), whereas others reported enhanced V1 response (Brieber et al., 2010). Specifically, our current results are at odds with two previous studies that measured responses to partially-coherent moving dots displays: Brieber et al. (2010) reported enhanced responses in ASD in both V1 and MT+, whereas Robertson et al. (2014) reported reduced responses in both regions. However, as highlighted by the latter study, differences in behavior as well as in neural circuits in ASD are specific to certain experimental conditions and stimulus properties. Robertson et al. (2014) demonstrated group differences when the duration of stimuli presentation is short and there is limited information to form a global percept, but not when stimuli are presented for a longer period of time. Additionally, Robertson et al. (2014) and Brieber et al. (2010), as well as others (Manning et al., 2015) show that group differences depend on coherence of the motion signal. Other factors that have been found to modulate group differences in motion perception include speed (Manning et al., 2013) and stimulus size (Schauder et al., 2017). Thus, inconsistency in imaging findings could be related to the choice of motion stimuli and experimental details in the fMRI paradigms. Our current findings highlight further challenges in characterizing visual processing changes in ASD. First, the effects in this study depended on retinotopic location. If, for example, we had placed the stimuli in more traditional peripheral locations, as commonly done in visual neuroscience studies using fMRI, and specifically in previous ASD studies (Dinstein et al., 2012; Robertson et al., 2014; Haigh et al., 2015), we would not have observed any group differences. Furthermore, the taskrelevance of the motion signals is a potentially important issue, and its effect on neural processing is yet to be assessed. The subtlety and specificity of visual processing differences in ASD may yield mechanistic insights as to their origin, but also render them hard to detect and make it difficult to generalize across paradigms and studies.

Second, it is important to note that the effect sizes reported in this study are small to modest. An inspection of individual sub- 
jects' data reveal that only a subset of the ASD group demonstrate the pattern of attenuated V1 and enhanced MT+ (Fig. 2, scatter plot). Given the general heterogeneity of ASD, in clinical presentation, behavior, and genetics (Jeste and Geschwind, 2014; Chaste et al., 2015; Bedford et al., 2019; Lombardo et al., 2019), such individual differences in neural response pattern are unsurprising. However, it implies that such effects are highly susceptible to random differences between study samples, especially with small sample sizes (Haigh et al., 2015). Overall, our results suggest that characterizing differences in cortical circuitry in ASD likely requires nuanced, individual-differences based approaches with larger sample sizes.

Our findings of differential response magnitude at different stages of processing in the visual pathway are in line with recent work in mouse models of ASD, indicating complex patterns of $\mathrm{E} / \mathrm{I}$ imbalance. Although the original E/I model suggested brainwide alteration of $\mathrm{E} / \mathrm{I}$ ratio that leads to an increase in neuronal excitability, recent findings from animal work show that increases in $\mathrm{E} / \mathrm{I}$ at the synaptic level do not necessarily imply higher firing rates, i.e., higher excitability (Antoine et al., 2019). It has also been shown that $\mathrm{E} / \mathrm{I}$ imbalance is region-specific (Gonçalves et al., 2017), and that $\mathrm{E} / \mathrm{I}$ balance changes throughout development and can even flip direction (O'Donnell et al., 2017). These findings suggest that the basic E/I model should be expanded and finetuned to capture the complex dynamics of E/I. Our results extend this claim to human neuroimaging, suggesting that future models of $\mathrm{E} / \mathrm{I}$ should account for the structure of functional pathways in the brain and the possibility that ASD involves altered corticocortical network interactions.

\section{References}

American Psychiatric Association (2013) Diagnostic and statistical manual of mental disorders (DSM-5). Arlington, VA: American Psychiatric Association.

Angelucci A, Bressloff PC (2006) Contribution of feedforward, lateral and feedback connections to the classical receptive field center and extraclassical receptive field surround of primate V1 neurons. Prog Brain Res 154:93-120.

Anliker J (1976) Eye movements: online measurement, analysis, and control. In: Eye movements and psychological processes (Monty RA, Senders JW, eds), pp 185-189. Hillsdale, NJ: Routledge.

Antoine MW, Langberg T, Schnepel P, Feldman DE (2019) Increased excitation-inhibition ratio stabilizes synapse and circuit excitability in four autism mouse models. Neuron 101:648-661.e4.

Bair W, Cavanaugh JR, Movshon JA (2003) Time course and time-distance relationships for surround suppression in macaque V1 neurons. J Neurosci 23:7690-7701.

Bedford SA, Park MT, Devenyi GA, Tullo S, Germann J, Patel R, Anagnostou E, Baron-Cohen S, Bullmore ET, Chura LR, Craig MC, Ecker C, Floris DL, Holt RJ, Lenroot R, Lerch JP, Lombardo MV, Murphy DGM, Raznahan A, Ruigrok ANV, et al. (2019) Large-scale analyses of the relationship between sex, age and intelligence quotient heterogeneity and cortical morphometry in autism spectrum disorder. Mol Psychiatry. Advance online publication. Retrieved April 26, 2019. doi:10.1038/s41380-0190420-6.

Behzadi Y, Restom K, Liau J, Liu TT (2007) A component based noise correction method (CompCor) for BOLD and perfusion based fMRI. Neuroimage 37:90-101.

Blignaut P (2009) Fixation identification: the optimum threshold for a dispersion algorithm. Atten Percept Psychophys 71:881-895.

Boynton GM, Demb JB, Glover GH, Heeger DJ (1999) Neuronal basis of contrast discrimination. Vision Res 39:257-269.

Brieber S, Herpertz-Dahlmann B, Fink GR, Kamp-Becker I, Remschmidt H, Konrad K (2010) Coherent motion processing in autism spectrum disorder (ASD): an fMRI study. Neuropsychologia 48:1644-1651.

Bullier J (2001) Feedback connections and conscious vision. Trends Cogn Sci 5:369-370.
Bullier J, Hupé JM, James A, Girard P (1996) Functional interactions between areas V1 and V2 in the monkey. J Physiol Paris 90:217-220.

Butler JS, Molholm S, Andrade GN, Foxe JJ (2017) An examination of the neural unreliability thesis of autism. Cereb cortex 27:185-200.

Chambers CD, Allen CP, Maizey L, Williams MA (2013) Is delayed foveal feedback critical for extra-foveal perception? Cortex 49:327-335.

Chaste P, Klei L, Sanders SJ, Hus V, Murtha MT, Lowe JK, Willsey AJ, Moreno-De-Luca D, Yu TW, Fombonne E, Geschwind D, Grice DE, Ledbetter DH, Mane SM, Martin DM, Morrow EM, Walsh CA, Sutcliffe JS, Lese Martin C, Beaudet AL, et al. (2015) A genome-wide association study of autism using the Simons simplex collection: does reducing phenotypic heterogeneity in autism increase genetic homogeneity? Biol Psychiatry 77:775-784.

Chen N, Lu J, Shao H, Weng X, Fang F (2017) Neural mechanisms of motion perceptual learning in noise. Hum Brain Mapp 38:6029-6042.

Chen Y, Norton D, McBain R, Gold J, Frazier JA, Coyle JT (2012) Enhanced local processing of dynamic visual information in autism: evidence from speed discrimination. Neuropsychologia 50:733-739.

Contractor A, Klyachko VA, Portera-Cailliau C (2015) Altered neuronal and circuit excitability in fragile $\mathrm{X}$ syndrome. Neuron 87:699-715.

de Jonge MV, Kemner C, de Haan EH, Coppens JE, van den Berg T, van Engeland H (2007) Visual information processing in high-functioning individuals with autism spectrum disorders and their parents. Neuropsychology 21:65-73.

Dickinson A, Jones M, Milne E (2016) Measuring neural excitation and inhibition in autism: different approaches, different findings and different interpretations. Brain Res 1648:277-289.

Dinstein I, Heeger DJ, Lorenzi L, Minshew NJ, Malach R, Behrmann M (2012) Unreliable evoked responses in autism. Neuron 75:981-991.

Esteban O, Markiewicz CJ, Blair RW, Moodie CA, Isik AI, Erramuzpe A, Kent JD, Goncalves M, DuPre E, Snyder M, Oya H, Ghosh SS, Wright J, Durnez J, Poldrack RA, Gorgolewski KJ (2019) fMRIPrep: a robust preprocessing pipeline for functional MRI. Nat Methods 16:111-116.

Fan X, Wang L, Shao H, Kersten D, He S (2016) Temporally flexible feedback signal to foveal cortex for peripheral object recognition. Proc Natl Acad Sci U S A 113:11627-11632.

Felleman DJ, Van Essen DC (1991) Distributed hierarchical processing in the primate cereb cortex. Cereb Cortex 1:1-47.

Foss-Feig JH, Tadin D, Schauder KB, Cascio CJ (2013) A substantial and unexpected enhancement of motion perception in autism. J Neurosci 33:8243-8249.

Gainey MA, Feldman DE (2017) Multiple shared mechanisms for homeostatic plasticity in rodent somatosensory and visual cortex. Philos Trans R Soc Lond B Biol Sci 372:20160157.

Gilbert CD, Li W (2013) Top-down influences on visual processing. Nat Rev Neurosci 14:350-363.

Gogolla N, Leblanc JJ, Quast KB, Südhof TC, Fagiolini M, Hensch TK (2009) Common circuit defect of excitatory-inhibitory balance in mouse models of autism. J Neurodev Disord 1:172-181.

Gonçalves J, Violante IR, Sereno J, Leitão RA, Cai Y, Abrunhosa A, Silva AP, Silva AJ, Castelo-Branco M (2017) Testing the excitation/inhibition imbalance hypothesis in a mouse model of the autism spectrum disorder: in vivo neurospectroscopy and molecular evidence for regional phenotypes. Mol Autism 8:47.

Haigh SM, Heeger DJ, Dinstein I, Minshew N, Behrmann M (2015) Cortical variability in the sensory-evoked response in autism. J Autism Dev Disord 45:1176-1190.

Han S, Tai C, Westenbroek RE, Yu FH, Cheah CS, Potter GB, Rubenstein JL, Scheuer T, de la Iglesia HO, Catterall WA (2012) Autistic-like behaviour in Scnla ${ }^{+/-}$mice and rescue by enhanced GABA-mediated neurotransmission. Nature 489:385-390.

Heeger DJ (2017) Theory of cortical function. Proc Natl Acad Sci U S A 114:1773-1782.

Herrington JD, Baron-Cohen S, Wheelwright SJ, Singh KD, Bullmore ET, Brammer M, Williams SC (2007) The role of MT+/V5 during biological motion perception in Asperger syndrome: an fMRI study. Res Autism Spectr Disord 1:14-27.

Huk AC, Dougherty RF, Heeger DJ (2002) Retinotopy and functional subdivision of human areas MT and MST. J Neurosci 22:7195-7205.

Hupé JM, James A, Payne B, Lomber S, Girard P, Bullier J (1998) Cortical feedback improves discrimination between figure and background by V1, V2 and V3 neurons. Nature 394:784-787. 
Jeste SS, Geschwind DH (2014) Disentangling the heterogeneity of autism spectrum disorder through genetic findings. Nat Rev Neurol 10:74-81.

Koh HC, Milne E, Dobkins K (2010a) Spatial contrast sensitivity in adolescents with autism spectrum disorders. J Autism Dev Disord 40:978-987.

Koh HC, Milne E, Dobkins K (2010b) Contrast sensitivity for motion detection and direction discrimination in adolescents with autism spectrum disorders and their siblings. Neuropsychologia 48:4046-4056.

Koldewyn K, Whitney D, Rivera SM (2011) Neural correlates of coherent and biological motion perception in autism. Dev Sci 14:1075-1088.

Lee E, Lee J, Kim E (2017) Excitation/inhibition imbalance in animal models of autism spectrum disorders. Biol Psychiatry 81:838-847.

Lombardo MV, Lai MC, Baron-Cohen S (2019) Big data approaches to decomposing heterogeneity across the autism spectrum. Mol Psychiatry 24:1435-1450.

Lord C, Bishop S (2015) Recent advances in autism research as reflected in dsm-5 criteria for autism spectrum disorder. Annu Rev Clin Psychol 11:53-70.

Lord C, Rutter M, Le Couteur A (1994) Autism diagnostic interview-revised: a revised version of a diagnostic interview for caregivers of individuals with possible pervasive developmental disorders. J Autism Dev Disord 24:659-685.

Lord C, Rutter M, DiLavore PC, Risi S, Gotham K, Bishop S (2012) Autism diagnostic observation schedule: ADOS. Los Angeles: Western Psychological Services.

Manning C, Charman T, Pellicano E (2013) Processing slow and fast motion in children with autism spectrum conditions. Autism Res 6:531-541.

Manning C, Tibber MS, Charman T, Dakin SC, Pellicano E (2015) Enhanced integration of motion information in children with autism. J Neurosci 35:6979-6986.

Maunsell JH, van Essen DC (1983) The connections of the middle temporal visual area (MT) and their relationship to a cortical hierarchy in the macaque monkey. J Neurosci 3:2563-2586.

Mejias JF, Murray JD, Kennedy H, Wang XJ (2016) Feedforward and feedback frequency-dependent interactions in a large-scale laminar network of the primate cortex. Sci Adv 2:e1601335.

Millin R, Kolodny T, Flevaris AV, Kale AM, Schallmo MP, Gerdts J, Bernier RA, Murray S (2018) Reduced auditory cortical adaptation in autism spectrum disorder. eLife 7:e36493.

Milne E (2011) Increased intra-participant variability in children with autistic spectrum disorders: evidence from single-trial analysis of evoked EEG. Front Psychol 2:51.

Milne E, Swettenham J, Hansen P, Campbell R, Jeffries H, Plaisted K (2002) High motion coherence thresholds in children with autism. J Child Psychol Psychiatry 43:255-263.

Muckli L, Kohler A, Kriegeskorte N, Singer W (2005) Primary visual cortex activity along the apparent-motion trace reflects illusory perception. PLoS Biol 3:e265.

Murray SO, Schallmo MP, Kolodny T, Millin R, Kale A, Thomas P, Rammsayer TH, Troche SJ, Bernier RA, Tadin D (2018) Sex differences in visual motion processing. Curr Biol 28:2794-2799.e3.

Nelson SB, Valakh V (2015) Excitatory/inhibitory balance and circuit homeostasis in autism spectrum disorders. Neuron 87:684-698.

O’Donnell C, Tiago Gonçalves JT, Portera-Cailliau C, Sejnowski TJ (2017) Beyond excitation/inhibition imbalance in multidimensional models of neural circuit changes in brain disorders. eLife 6:e26724.

Pascual-Leone A, Walsh V (2001) Fast backprojections from the motion to the primary visual area necessary for visual awareness. Science 292:510-512.

Pellicano E, Gibson L, Maybery M, Durkin K, Badcock DR (2005) Abnormal global processing along the dorsal visual pathway in autism: a possible mechanism for weak visuospatial coherence? Neuropsychologia 43:10441053.

Power JD, Mitra A, Laumann TO, Snyder AZ, Schlaggar BL, Petersen SE (2014) Methods to detect, characterize, and remove motion artifact in resting state fMRI. Neuroimage 84:320-341.

Ramocki MB, Zoghbi HY (2008) Failure of neuronal homeostasis results in common neuropsychiatric phenotypes. Nature 455:912-918.
Ress D, Heeger DJ (2003) Neuronal correlates of perception in early visual cortex. Nat Neurosci 6:414-420.

Robertson CE, Martin A, Baker CI, Baron-Cohen S (2012) Atypical integration of motion signals in autism spectrum conditions. PLoS One 7:e48173.

Robertson CE, Thomas C, Kravitz DJ, Wallace GL, Baron-Cohen S, Martin A, Baker CI (2014) Global motion perception deficits in autism are reflected as early as primary visual cortex. Brain 137:2588-2599.

Rubenstein JL, Merzenich MM (2003) Model of autism: increased ratio of excitation/inhibition in key neural systems. Genes Brain Behav 2:255-267.

Schallmo MP, Kale AM, Millin R, Flevaris AV, Brkanac Z, Edden RA, Bernier RA, Murray SO (2018) Suppression and facilitation of human neural responses. eLife 7:e30334.

Schallmo MP, Kolodny T, Kale AM, Millin R, Flevaris AV, Edden RA, Gerdts J, Bernier RA, Murray SO (2019a) Weaker neural suppression in autism. bioRxiv. doi:10.1101/645846.

Schallmo MP, Millin R, Kale AM, Kolodny T, Edden RAE, Bernier RA, Murray SO (2019b) Glutamatergic facilitation of neural responses in MT enhances motion perception in humans. Neuroimage 184:925-931.

Schauder KB, Park WJ, Tadin D, Bennetto L (2017) Larger receptive field size as a mechanism underlying atypical motion perception in autism spectrum disorder. Clin Psychol Sci 5:827-842.

Schira MM, Tyler CW, Breakspear M, Spehar B (2009) The foveal confluence in human visual cortex. J Neurosci 29:9050-9058.

Shipp S, Zeki S (1989) The organization of connections between areas V5 and V1 in macaque monkey visual cortex. Eur J Neurosci 1:309-332.

Siegel JS, Power JD, Dubis JW, Vogel AC, Church JA, Schlaggar BL, Petersen SE (2014) Statistical improvements in functional magnetic resonance imaging analyses produced by censoring high-motion data points. Hum Brain Mapp 35:1981-1996.

Sillito AM, Cudeiro J, Jones HE (2006) Always returning: feedback and sensory processing in visual cortex and thalamus. Trends Neurosci 29:307-316.

Silvanto J, Lavie N, Walsh V (2005) Double dissociation of V1 and V5/MT activity in visual awareness. Cereb Cortex 15:1736-1741.

Spencer J, O’Brien J, Riggs K, Braddick O, Atkinson J, Wattam-Bell J (2000) Motion processing in autism: evidence for a dorsal stream deficiency. Neuroreport 11:2765-2767.

Stano JF (2004) Wechsler abbreviated scale of intelligence. Rehabil Couns Bull 48:56-57.

Sysoeva OV, Galuta IA, Davletshina MS, Orekhova EV, Stroganova TA (2017) Abnormal size-dependent modulation of motion perception in children with autism spectrum disorder (ASD). Front Neurosci 11:164.

Tavassoli T, Latham K, Bach M, Dakin SC, Baron-Cohen S (2011) Psychophysical measures of visual acuity in autism spectrum conditions. Vision Res 51:1778-1780.

Turkozer HB, Pamir Z, Boyaci H (2016) Contrast affects fMRI activity in middle temporal cortex related to center-surround interaction in motion perception. Front Psychol 7:454.

Turrigiano G (2011) Too many cooks? Intrinsic and synaptic homeostatic mechanisms in cortical circuit refinement. Annu Rev Neurosci 34:89-103.

Turrigiano G (2012) Homeostatic synaptic plasticity: local and global mechanisms for stabilizing neuronal function. Cold Spring Harb Perspect Biol 4:a005736.

Ungerleider LG, Desimone R (1986) Cortical connections of visual area MT in the macaque. J Comp Neurol 248:190-222.

Vadillo MA, Street CNH, Beesley T, Shanks DR (2015) A simple algorithm for the offline recalibration of eye-tracking data through best-fitting linear transformation. Behav Res Methods 47:1365-1376.

Williams MA, Baker CI, Op de Beeck HP, Shim WM, Dang S, Triantafyllou C, Kanwisher N (2008) Feedback of visual object information to foveal retinotopic cortex. Nat Neurosci 11:1439-1445.

Yizhar O, Fenno LE, Prigge M, Schneider F, Davidson TJ, O'Shea DJ, Sohal VS, Goshen I, Finkelstein J, Paz JT, Stehfest K, Fudim R, Ramakrishnan C, Huguenard JR, Hegemann P, Deisseroth K (2011) Neocortical excitation/inhibition balance in information processing and social dysfunction. Nature 477:171-178. 